Working Paper 93-05

Statistics and Econometrics Series 05

February 1993
Departamento de Estadística y Econometría

Universidad Carlos III de Madrid

Calle Madrid, 126

28903 Getafe (Spain)

Fax (341) 624-9849

\title{
BOOTSTRAPPING THE GENERAL LINEAR HYPOTHESIS TEST
}

Pedro Delicado and Manuel del Río*

\begin{abstract}
We discuss the use of bootstrap methodology in hypothesis testing, focusing on the classical F-test for linear hypotheses in the linear model. A modification of the F-statistics which allows for resampling under the null hypothesis is proposed. This approach is specifically considered in the one-way analysis of variance model. A simulation study illustrating the behaviour of our proposal is presented.
\end{abstract}

Key Words

Bootstrap, F-test; General Linear Hypothesis; Hypothesis Testing; Linear Model; One-way Model; Resampling.

*Delicado, Departamento de Estadística y Econometría, Universidad Carlos III de Madrid; Del Río, Departamento de Estadística e Investigación Operativa, Universidad Complutense de Madrid. Correspondence to: Manuel del Rio, Departamento Estadística e Investigación Operativa, Facultad de Matemáticas, Universidad Complutense de Madrid, 28040 Madrid, Spain. 
) 


\section{Introduction.}

The use of bootstrap methodology in hypothesis testing -essentially based on approximating the critical values by bootstrap distributions - has received less attention that its application in other problems like, for instance, the construction of confidence regions. Here are some recent references. Beran (1988) studies the asymptotic error in level of bootstrap tests and the improvement given by prepivoting the test statistic. In Hinkley (1988) some general ideas about bootstrap testing are briefly discussed. Romano (1988) uses the bootstrap to aproximate critical values of nonparametric tests based on measures defined on the empirical distribution. Hall and Wilson (1991) and Hal! (1912) insist in two guidelines that we analyze in Section 2: usage of pivotal functions and resampling reflecting the null hypothesis. In Mammen (1992) the convergence of bootstrapped F-test in linear models is proved.

This paper is concerned with the use of bootstrap idea in hypothesis testing. Section 3 is dedicated to present our work for testing a general linear hypothesis in a linear model. Following the second guideline cited above, we propose a natural modification of the classical F-statistics that permits resampling under the null hypothesis, though the suta fail to comply with it. In Section 4, this proposal -and the basic idea of resampling taking into account the model in consideration- is analyzed with some detail in the framework of the one-way analysis of variance. ln Section 5 , the corresponding behaviour is illustrated by reporting the results of a simulation study.

\section{Two general guidelines.}

Naturally, the duality between hypothesis testing and confidence regions is maintained under bootstrap methodology. That leads to consider the first guideline cited: use of (asymptotically) pivotal quantities, that could be extended to the use of methods with good behaviour in the problem of confidence interval construction. In general, acting in this way will imply an improvement in the test level. 
However, there is an important conceptual difference when both problems are considered under the bootstrap approach. In hypothesis testing accurate estimates of the critical values are needed, even if the data had their origin in the alternative hypothesis. This demand could invalidate some direct approximations to the distribution of interest. Consider, as in Hall and Wilson (1991), a one-dimensional parameter situation and the simple testing problem $H_{0}: \theta=\theta_{0}$ against $H_{1}: \theta \neq \theta_{0}$. Given that $T=T\left(X_{1}, \ldots, X_{n}\right)$ is a good estimator of the unknown $\theta$, a reasonable test could be based on the difference $T-\theta_{0}$. If our goal is in relation to its level or p-value, our interest lies in the distribution of $T-\theta_{0}$ under $H_{0}$. A direct -and naïve- application of the bootstrap method would lead to estimate this distribution using the statistic $T^{*}-\theta_{0}$, where $T^{*}$ is the value of $T$ in the resampling, that is, under the empirical distribution of $\left(X_{1}, \ldots, X_{n}\right)$. In Hall $(1992, \operatorname{Sec} .3 .12)$ it is shown the bad behaviour of the power function of the corresponding test. The trouble here is that $T^{*}-\theta_{0}$ does not approximate the null hypothesis when the sample comes from a parameter far away from $\theta_{0}$.

Hall and Wilson (1991) propose to estimate the $T-\theta_{0}$ distribution by means of the statistic $T^{*}-T$. Note that this corresponds to resampling the quantity $T_{0}=T-\theta$ instead of $T-\theta_{0}$. In the next section we extend this idea to testing a general linear hypothesis in a linear model.

\section{Bootstrap hypothesis testing in the linear model.}

lt will be convenient to consider the coordinate-free version of the linear model. Let $Y=\mu+\epsilon$, where $Y$ denotes the n-dimensional vector of observations, $\mu$ is the vector of means belonging to the subspace $V \subset \mathbf{R}^{n}$ with dimension $p<n$, and $e$ is the vector of independent errors $e_{i} \sim F$ such that $E(e)=0$. We want to test $H_{0}: \mu \in V_{0}$ versus $\mu \notin V_{0}$. being $V_{0} \subset V$ a subspace with dimension $p_{0}<p$. The usual F-statistic can be written as 


$$
T=T(Y)=\frac{\left\|\mathbf{P}_{V \mid V_{0}} Y\right\|^{2} /\left(p-p_{0}\right)}{\left\|\mathbf{P}_{V \perp} Y\right\|^{2} /(n-p)}
$$

where $V^{\perp}, V \mid V_{0}$, and $\mathbf{P}_{W}$ denote, respectively, the subspace orthogonal to $\mathrm{V}$, the orthogonal complement of $V_{0}$ in $V$, and the orthogonal projection onto $W$.

Here is an schematic outline of the bootstrap procedure for this version of the linear model. We start from the model $(\mu, F)$; after observing the response vector $Y$, we adjust $\left(\hat{\mu}, \hat{F}_{n}\right)$, being $\hat{\mu}=\mathbf{P}_{V} Y$ and $\hat{F}_{n}$ the empirical distribution of the residual vector $\hat{e}=Y-\hat{\mu}=\mathbf{P}_{V \perp} Y$, that we suppose centered at 0 . The bootstrap estimation of an arbitrary function $R=R(Y, F)$ is the (conditional) distribution of $R^{*}=R\left(Y^{*}, \hat{F}_{n}\right)$ where $Y^{*}=\hat{\mu}+e^{*}$, and the vector $e^{*}$ has independent components $e_{i}^{*} \sim \hat{F}_{n}$.

The bootstrap methodology proposes to consider as the critical region of nominal $\alpha$-level $T>\hat{t}_{\alpha}$, where $\hat{t}_{\alpha}$ is the $1-\alpha$ quantile of the bootstrap distribution of $\mathrm{T}$ under samples coming from $H_{0}$, i.e., $P\left(T\left(Y^{*}\right) \leq \hat{t}_{\alpha} \mid Y \in H_{0}\right)=1-\alpha$.

Hence, we need to know - or, in the practice, to approximate by Monte Carlo trials- this null bootstrap distribution. For this, we propose to consider

$$
T_{0}=T_{0}(Y, \mu)=\frac{\left\|\mathbf{P}_{V \mid V_{0}}(Y-\mu)\right\|^{2} /\left(p-p_{0}\right)}{\left\|\mathbf{P}_{V^{\perp}}(Y-\mu)\right\|^{2} /(n-p)}=\frac{\left\|\mathbf{P}_{V \mid V_{0}} e\right\|^{2} /\left(p-p_{0}\right)}{\left\|\mathbf{P}_{V \perp} e\right\|^{2} /(n-p)} .
$$

Comments:

i) Since it is based on $Y-\mu, T_{0}$ does not relies on the hypothesis under which the data are obtained; in other words, it is invariant against the hypothesis generating the data. Besides, $T_{0}$ agrees with $T$ under $H_{0}$.

ii) A direct reasoning leading to $T_{0}$ is the following. Since we attempt to approximate the null distribution of $T$, let us take an arbitrary $\mu_{0} \in V$ and transform $Y$ to $Y_{0}=Y-\mu+\mu_{0}$. The vector $Y_{0}$ verifies $H_{0}$ and, we are naturally conducted to

$$
T\left(Y_{0}\right)=\frac{\left\|\mathbf{P}_{V \mid V_{0}} Y_{0}\right\|^{2} /\left(p-p_{0}\right)}{\left\|\mathbf{P}_{V^{\perp}} Y_{0}\right\|^{2} /(n-p)}=\frac{\left\|\mathbf{P}_{V \mid V_{0}}(Y-\mu)\right\|^{2} /\left(p-p_{0}\right)}{\left\|\mathbf{P}_{V^{\perp}}(Y-\mu)\right\|^{2} /(n-p)}=T_{0}(Y, \mu) .
$$

Note that the above expression is independent of the initial vector $\mu_{0} \in V$. 
Therefore, the bootstrap distribution of $T_{0}(Y, \mu)$ is the null bootstrap distribution of $T$; consequently, the critical value $\hat{t}_{\alpha}$ should be taken verifying

$$
P\left(T_{0}^{*}=T_{0}\left(Y^{*}, \mu\right) \leq \hat{t}_{\alpha} \mid Y\right)=1-\alpha
$$

We see that the modification of the F-statistics arises in a natural and direct way. In some sense, this presentation completes the one given in Mammen (1992). Moreover, his results assure the convergence of our proposal.

\section{Bootstrapping the one-way ANOVA model.}

We now consider the previous proposal in the particular case of testing equality of means in the one-way model.

\subsection{Raising the problem.}

We assume a set of $n_{i}$ observations, $Y_{i j}, j=1, \ldots, n_{i}$, coming from a population $P_{i}$ with mean $\mu_{i}$ and variance $\sigma^{2}, i=1, \ldots, p$. We want to test $H_{0}: \mu_{1}=$ $\cdots=\mu_{p}$. This problem fits into the framework of last section by defining $Y=$ $\left(Y_{11}, \ldots, Y_{1 n_{1}}, \ldots, Y_{p_{n}}\right)^{\prime}, \quad n=\sum_{i=1}^{p} n_{i}, \mu=\left(\mu_{1}, \ldots, \mu_{p}\right)^{\prime} ; V_{0}$ and $V$ are now the spaces spanned, respectively, by the vector $\left.1_{n}=(1, . .2), 1\right)^{\prime}$ and by the $p$ vectors in $\mathbb{R}^{n}:\left(1_{n_{1}}^{\prime}, 0\right)^{\prime},\left(0,1_{n_{2}}^{\prime}, 0\right)^{\prime}, \ldots,\left(0,1_{n_{p}}^{\prime}\right)^{\prime}$. It is well know that

$$
T(Y)=\frac{\sum_{i} n_{i}\left(\bar{Y}_{i}-\bar{Y}\right)^{2} /(p-1)}{\sum_{i} \sum_{j}\left(Y_{i j}-\bar{Y}_{i}\right)^{2} /(n-p)}
$$

The statistic $T_{0}$ in $(3.2)$ is now

$$
T_{0}(Y, \mu)=\frac{\sum_{i} n_{i}\left(\bar{X}_{i}-\bar{X}\right)^{2} /(p-1)}{\sum_{i} \sum_{j}\left(X_{i, j}-\bar{X}_{i}\right)^{2} /(n-p)}=\frac{\sum_{i} n_{i}\left(\bar{e}_{i}-\bar{e}\right)^{2} /(p-1)}{\sum_{i} \sum_{j}\left(e_{i j}-\bar{e}_{i}\right)^{2} /(n-p)},
$$

where $X_{i j}=Y_{i j}-\mu_{i}=e_{i j}, \bar{X}_{i}=\sum_{j} X_{i j} / n_{i}, \bar{X}=\sum_{i} \sum_{j} X_{i j} / n$. 


\subsection{Resampling schemes.}

To obtain a good approximation through the bootstrap approach in a general arbitrary situation, the resampling distribution must reflect the model distribution in an adequate way. In our concrete situation this means that the bootstrap distribution of $T_{0}$ must be obtained "mimicking" the considered model. The one-way model will allow us to illustrate this comment by considering two slightly different sets of assumptions on the underlying populations.

\section{Resampling $R_{1}$ : Identical populations.}

This is the standard case where the distributions associated to the populations differ only in the means, that $i \therefore \epsilon_{j} \sim F_{i}=F, i=1, \ldots, p$. Since errors are interchangeable through the populations, we should take $\hat{F}_{n}$ as the empirical distribution of the $n$ residuals $\hat{e}_{i j}=Y_{i j}-\bar{Y}_{i}, i=1, \ldots, p, j=1, \ldots, n_{i}$. Recalling the schematic outline presented after (3.1), we have

$$
T_{0}^{*}=\frac{\sum_{i} n_{i}\left(\bar{X}_{i}^{*}-\bar{X}^{*}\right)^{2} /(p-1)}{\sum_{i} \sum_{j}\left(X_{i j}^{*}-\bar{X}_{i}^{*}\right)^{2} /(n-p)}=\frac{\sum_{i} n_{i}\left(\bar{e}_{i}^{*}-\bar{e}^{*}\right)^{2} /(p-1)}{\sum_{i} \sum_{j}\left(e_{i j}^{*}-\bar{e}_{i}^{*}\right)^{2} /(n-p)}
$$

where $X_{i j}^{*}=Y_{i j}^{*}-\hat{\mu}_{i}=e_{i j}^{*} \bar{Y}_{i}-\bar{Y}_{i}=e_{i j}^{*} \sim \hat{F}_{n}$.

In practice, the simulation of the $T_{0}^{*}$ distribution will be done generating $B$ independent samples from the empirical distribution of the $n$ residuals $\hat{e}_{i j}$, that is, considering $B$ sets with $n$ elements chosen with replacement from $R=\left\{\hat{e}_{i j}, i=1, \ldots, p, j=\right.$ $\left.1, \ldots, n_{i}\right\}$.

\section{Resampling $R_{2}$ : Different populations.}

Assume now the distributions associated with every population are different not only in the means but in other aspects; that is, $e_{i j}$ are independent and $e_{i j} \sim F_{i}, j=$ $1, \ldots, n_{i}$, where $F_{i}$ are different with mean 0 and variance $\sigma^{2}, i=1, \ldots, p$. To reflect the new joint distribution of the vector of errors, we will take now $\hat{F}_{n}$ as the joint distribution of the $p$ empirical independent distributions, $\hat{F}_{i n_{i}}$, corresponding to the sets $R_{i}=\left\{\hat{\epsilon}_{i j}, j=1, \ldots, n_{i}\right\}, i=1, \ldots, p$. The statistic $T_{0}^{*}$ still has the expresion 
(4.3), with $X_{i j}^{*}=e_{i j}^{*} \sim \hat{F}_{i n_{i}}$.

In practice, $T_{0}^{*}$ will be simulated from $B$ sets of $n$ elements, $n_{i}$ of them will be selected with replacement from $R_{i}, i=1, \ldots, p$. Note that resampling from $R_{i}$ is equivalent to resampling from $O_{i}=\left\{Y_{i j}, j=1, \ldots, n_{i}\right\}$, then obtaining $O_{i}^{*}=\left\{Y_{i j}^{*}, j=\right.$ $\left.1, \ldots, n_{i}\right\}$, and, finally, defining $X_{i j}^{*}=Y_{i j}^{*}-\bar{Y}_{i}, j=1, \ldots, n_{i}$.

\subsection{Normalizing the residuals.}

The idea of reflecting the model hypothesis in the bootstrap scheme, leads us to consider the resampling in a population of residuals where the empirical variances in each subpopulation are equal; without loss of generality we take this common value equal to 1 . This leads us to replace $(4.3)$ by

$$
T_{N}^{*}=\frac{\sum_{i} n_{i}\left(\bar{e}_{N i}^{*}-\bar{\epsilon}_{N}^{*}\right)^{2} /(p-1)}{\sum_{i} \sum_{j}\left(e_{N i j}^{*}-\bar{\epsilon}_{N i}^{*}\right)^{2} /(n-p)},
$$

where $e_{N i j}^{*} \sim \hat{F}_{N n}$, and $\hat{F}_{N n}^{*}$ is the empirical (joint) distribution of the standardized residuals: $\hat{e}_{N i j}=\left(Y_{i j}-\bar{Y}_{i}\right) / \hat{\sigma}_{i}, \hat{\sigma}_{i}^{2}=\sum_{j}\left(Y_{i j}-\bar{Y}_{i}\right)^{2} / n_{i}$.

Note that the statistic $T_{N}^{*}$ can be also obtained in the following way. Assume initially heteroscedasticity and consider, as alternative to (4.2),

$$
T_{0 N}\left(Y, \mu, \sigma_{1}, \ldots, \sigma_{n}\right)=\frac{\sum_{i} n_{i}\left(\bar{W}_{i}-\bar{W}\right)^{2} /(p-1)}{\sum_{i} \sum_{j}\left(W_{i j}-\bar{W}_{i}\right)^{2} /(n-p)},
$$

where $W_{i j}=\left(Y_{i j}-\mu_{i}\right) / \sigma_{i}$, and $\sigma_{i}^{2}$ is the populational variance of $P_{i}$. If there exists homoscedasticity, $T_{0 N}$ becomes $T_{0}$ and its distribution is the same as the distribution of $T$ under $H_{0}$. It follows inmediately that the bootstrap distribution of $T_{0 N}^{*}$ coincides with the distribution of $T_{N}^{*}$. Heuristically, we should expect that this statistic will improve the approximation to the null distribution.

The simulation of $T_{N}^{*}$ will be done similarly as in Subsection 4.2., but starting with the normalized residuals $\hat{e}_{N i j}$. Both schemes above cited are still valid using now the normalized residuals. In the next section, we will denote them resampling $R_{3}$ and $R_{4}$. 


\section{A simulation study.}

In this section we illustrate the beliaviour of our proposal by means of a simulation study. The results, obtained using only 199 bootstrap resamples, generally indicate a satisfactory performance.

Through our simulation study, we have maintained three populations, i.e., $p=3$. We considered combinations of two nominal levels, $\alpha=.05, .1$, two total sample sizes, $n=30,60$, and three groups of error distributions associated to the populations, $G_{k}, k=1,2,3$. The composition of these groups is the following. In $G_{1}$ the errors have normal distributions; in $G_{2}$ they have shifted exponential distributions; and, in $G_{3}$ the errors have different distributions: $N(0,1)$, shifted exponential and normalized $t_{3}$.

The computations were performed using Fortran routines rumning on a DECstation $5000 / 200$ under Ultrix-32. The routines GGUBS, GGNML and GGAMR of the IMSL library are used to generate the pseudo-random variables and to make the resamplings.

\subsection{Results under the null hypothesis.}

Without loss of generality, we took $\mu_{1}=\mu_{2}=\mu_{3}=0$. The specific distributions for the above groups were: $N(0,1)$, shifted exponential with $\lambda=1$, and $t_{3}$ normalized to have variance unity.

We conducted 1000 trials under each of the 28 combinations of 4 particular population sizes and 7 types of resampling listed in Table 1 . In each trial, $B=199$ resamples were drawn from the empirical distribution associated to the resampling. By means of (3.3). these resamples provided the approximate $\hat{t}_{c x i}$ values and, in each trial, $H_{0}$ was rejected if $T(Y)>\hat{t}_{\alpha i}, \alpha=.05, .1, i=1, \ldots, 1000$. (Specifically, $\hat{t}_{\alpha i}$ was the 9th largest of the 199 values of $T_{0}^{*}$ when $\alpha=.05$, and the 19th largest when $\alpha=.1$.)

The entries of Table 1 are the bootstrap levels, $\alpha^{*}$, that is, the proportion of trials rejecting $H_{0}$. The symbol \# denotes cases with $\alpha^{*}$ levels differing significantly of $\alpha$. 


\begin{tabular}{|c|c|c|c|c|c|c|c|c|}
\hline \multirow{2}{*}{\multicolumn{2}{|c|}{$\begin{array}{c}\text { Error distributions } \\
\text { Resamplings }\end{array}$}} & \multicolumn{2}{|c|}{$G_{1}: N(0,1)$} & \multicolumn{2}{|c|}{$G_{2}: E(1)$} & \multicolumn{3}{|c|}{$G_{3}: N(0,1), E(1), t_{3}$} \\
\hline & & $R_{1}$ & $R_{3}$ & $R_{1}$ & $R_{3}$ & $R_{1}$ & $R_{2}$ & $R_{4}$ \\
\hline \multicolumn{9}{|c|}{ Population sizes } \\
\hline$(10,10,10)$ & $\alpha=.05$ & 0.0530 & 0.0550 & 0.0510 & 0.0480 & 0.0490 & $0.0320^{\#}$ & 0.0420 \\
\hline \multirow{3}{*}{$(5,10,15)$} & $\alpha=.1$ & 0.0990 & 0.0990 & 0.0950 & 0.0960 & 0.0980 & $0.0800^{\#}$ & 0.0960 \\
\hline & $\alpha=.05$ & 0.0380 & 0.0420 & 0.0440 & 0.0410 & 0.0600 & 0.0430 & 0.0520 \\
\hline & $\alpha=.1$ & 0.0910 & 0.0900 & 0.1090 & 0.1110 & 0.1150 & 0.0900 & 0.1100 \\
\hline \multirow[t]{2}{*}{$(20,20,20)$} & $\alpha=.05$ & 0.0430 & 0.0470 & 0.0460 & 0.0470 & 0.0500 & 0.0430 & 0.0500 \\
\hline & $\alpha=.1$ & 0.0960 & 0.0930 & 0.0970 & 0.0970 & 0.1120 & 0.0940 & 0.1050 \\
\hline \multirow[t]{2}{*}{$(10,20,30)$} & $\alpha=.05$ & 0.0460 & 0.0430 & 0.0570 & 0.0560 & 0.0630 & 0.0430 & 0.0520 \\
\hline & $\alpha=.1$ & 0.1010 & 0.0970 & 0.1100 & 0.1070 & 0.1060 & 0.0900 & 0.0970 \\
\hline
\end{tabular}

Table 1: Bootstrap levels for 28 combinations of error distributions, resamplings, and population sizes.

Figure 1 depicts the values of $\alpha^{*}$ for the last row of Table 1 as a function of the number of trials. The 40 points in the trajectories correspond to the values computed from each consecutive 25 trials.

Some comments are in order. Most of the bootstrap levels are very close to the nominal levels, specially when the appropriate resamplings are used, and even when the inadequate resamplig $R_{1}$ is used in $G_{3}$. The performances under normal or exponential distributed errors are very similar, pointing out that asymmetric errors are not troublesome. The stability of the trajectories is reached soon. For the group $G_{3}$ (different populations), the results with resampling $R_{2}$ are lightly better than with $R_{1}$ and both are dominated by the results using $R_{4}$.

(Insert Figure 1 about here) 


\subsection{Results under heteroscedasticity.}

Although our development does not deal with heteroscedastic situations, we planned to check how our proposal would perform under unequal variances. With this aim, we considered the groups $G_{1}$ and $G_{3}$. In $G_{1}$ the normal distributions had standard deviations $\sigma_{i}=i, i=1,2,3$; in $G_{3}$ we included standard normal, shifted exponential with $\sigma=2$, and $t_{3}(\sigma=3)$ distributions. The total sizes $(n=30,60)$ were assigned to the populations in three ways: balanced, higher sizes to higher variances, and viceversa. For each assignation, resamplings $R_{1}$ and $R_{2}$ were considered.

Both groups and sizes provided similar results. Figure 2 shows the 6 bootstrap level trajectories for $G_{1}$ and $n=30$. As a summary of empirical conclusions we have: the resampling $R_{1}$ was very unappropriated with unbalanced sizes, the resampling $R_{1}$ was beaten by $R_{2}$ in all the situations; therefore, if we suspect of possible heteroscedasticity, the resampling starting from different populations will provide more accurate levels. The best results occur when there is correspondence between sizes and variability in populations.

(Insert Figure 2 about here)

\subsection{Results under the alternative hypothesis.}

We have also verified the good approximation to the true critical values and the high power provided by the bootstrap test when the data come from populations with unequal means. Our illustration will use some of the combinations considered in Section 5.1 , being now $\mu_{1}=-1, \mu_{2}=0, \mu_{3}=1$.

\section{(lnsert Figure 3 about here)}

Figure 3 depicts box-plots (with whiskers ending at the extreme values) of 1000 bootstrap estimated critical values, $\hat{t}_{c i}$, obtained from (3.3). The errors belong to group $G_{1}$ with $N(0,1)$ and $G_{2}$ with shifted exponential, $\lambda=1$. (The true values are $F_{2,2 T, 05}=3.35 \mathrm{in} C_{1}$ and were obtained by simulation using 5000 trials in $\left(r_{2}.\right)$ 


\begin{tabular}{|cc|c|c|c|c|c|c|c|}
\hline \multicolumn{2}{|c|}{ Error distributions } & \multicolumn{2}{c|}{$G_{1}: N(0,1)$} & \multicolumn{2}{c|}{$G_{2}: E(1)$} & \multicolumn{2}{c|}{$G_{3}: N(0,1), E(1), t_{3}$} \\
\hline Resamplings & $R_{1}$ & $R_{3}$ & $R_{1}$ & $R_{3}$ & $R_{1}$ & $R_{2}$ & $R_{\mathbf{4}}$ \\
\hline \multicolumn{2}{|c|}{$\begin{array}{c}\text { Population sizes } \\
(10,10,10)\end{array}$} & & & &. & & & \\
& $\alpha=.05$ & 0.9720 & 0.9700 & 0.9550 & 0.9530 & 0.9680 & 0.9220 & 0.9520 \\
$(5,10,15)$ & $\alpha=.1$ & 0.9870 & 0.9860 & 0.9790 & 0.9760 & 0.9770 & 0.9560 & 0.9750 \\
\cline { 3 - 10 } & $\alpha=.1$ & 0.9370 & 0.9320 & 0.9250 & 0.9230 & 0.9160 & 0.8660 & 0.8920 \\
\cline { 3 - 9 }$(20,20,20)$ & $\alpha=.05$ & 1.0000 & 1.0000 & 1.0000 & 1.0000 & 0.9980 & 0.9930 & 0.9980 \\
& $\alpha=.1$ & 1.0000 & 1.0000 & 1.0000 & 1.0000 & 0.9980 & 0.9950 & 0.9980 \\
\cline { 3 - 9 }$(10,20,30)$ & $\alpha=.05$ & 1.0000 & 1.0000 & 0.9930 & 0.9950 & 0.9980 & 0.9910 & 0.9950 \\
& $\alpha=.1$ & 1.0000 & 1.0000 & 0.9970 & 0.9970 & 0.9980 & 0.9940 & 0.9970 \\
\hline
\end{tabular}

Table 2: Power in $H_{A}: \mu_{1}=-1, \mu_{2}=0, \mu_{3}=1$, for the 28 combinations of Table 1 .

Note the concentration around the true critical values, and the similar results given by $R_{1}$ and $R_{3}$.

Table 2 gives the proportion of trials rejecting the equality of means, that is, the power, under the above alternative for the combinations considered in Section 5.1. It is worthwhile noting the high values attained.

\subsection{Bootstrapping the F-statistic directly}

Finally, we will show the inaccuracy of the naive bootstrap directly based on the Fstatistic of (4.1), that is, when the resampling uses

$$
T_{D}^{*}=\frac{\sum_{i} n_{i}\left(\bar{Y}_{i}^{*}-\bar{Y}^{*}\right)^{2} /(p-1)}{\sum_{i} \sum_{j}\left(Y_{i j}^{*}-\bar{Y}_{i}^{*}\right)^{2} /(n-p)}
$$

where $Y_{i j}^{*}=e_{i j}^{*}+\bar{Y}_{i}$, and $e_{i, j}^{*}$ follow one of the empirical distributions presented in Sections 4.2 and 4.3 . We will refer this procedure as direct bootstrap.

We began conducting 1000 trials for the situation and combinations considered in Section 5.1. The direct bootstrap levels, $\alpha_{D}^{*}$, turned out to be much lower than the 
nominal levels -in fact, only 5 of 56 cases considered were different of zero with a maximum value of .004 . We also studied the distribution of the direct critical values, $\hat{t}_{\alpha i}^{D}, i=1, \ldots, 1000, \alpha=.05, .1$, corresponding to 4 combinations: groups $G_{1}$ and $G_{2}$ with resampling $R_{1}$, and $G_{3}$ with $R_{1}$ and $R_{3}$. The population sizes were $n_{i}=10$. This study was done with data from $H_{0}$ and from the alternative $H_{A}: \mu_{1}=-1, \mu_{2}=0, \mu_{3}=1$.

The distributions of the direct critical values were very similar in the four combinations. Figure 4 shows box-plots summarizing the distributions under $H_{0}$ and $H_{A}$, $\alpha=.05, n=30$, in the combination $G_{1}$ and $R_{1}$. For comparison, we have also included box-plots for the distributions of the critical values, $\hat{t}_{\alpha i}^{*}$, derived from our proposal.

\section{(lnsert Figure 4 about here)}

Two points clearly stand out. Whether the data came from $H_{0}$ or from $H_{A}$, the distribution of critical values $\hat{t}_{\alpha i}^{I)}$ is useless to approximate the true critical value $\left(F_{2,28,05}=3.35\right)$. On the contrary, both from $H_{0}$ and $H_{A}$, the distributions of proposed critical values $\hat{t}_{\alpha i}$ are extremely similar and very concentrated around the true critical value.

\section{References}

Beran, R., Prepivoting tests statistics: A bootstrap view of asymptotic refinements, J. Amer. Statist. Assoc., 83 (1988) 687-697.

Hall, P., The Bootstrap and Edgcworth Expansion (Springer-Verlag, New York, 1992)

Hall, P. and Wilson, S.R., Two guidelines for bootstrap hypothesis testing, Biometrics, 47 (1991) 757-762.

Hinkley, D. V., Bootstrap methods, J. Roy. Statist. Soc. Ser. B,50 (1988) 321-337.

Mammen, E., When does bootstrap work? (Lecture Notes in Statistics, Vol. 77, Springer-Verlag, New Y'ork, 1992)

Romano, J. H., A bootstrap revival of some nomparametric distance tests, J. Amer. Statist. Assoc., 83 (1988) 698-708. 


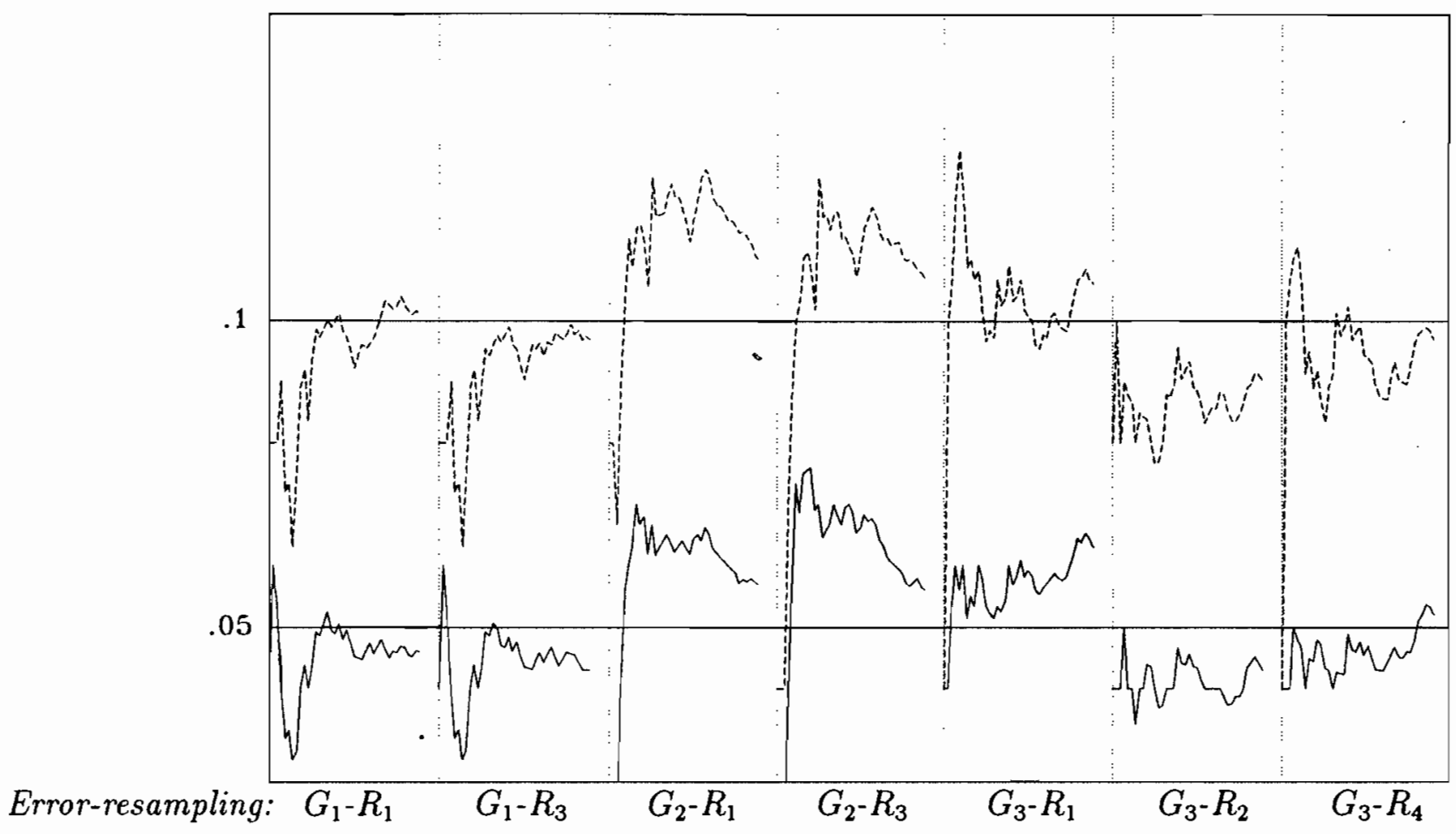

Fig. 1: Bootstrap levels as function of the number of trials $(\mathrm{N}=1000)$. The 40 points correspond to computations from each consecutive 25 trials.

$$
-\alpha=.05, \quad \cdots \alpha=.1 \text {. }
$$




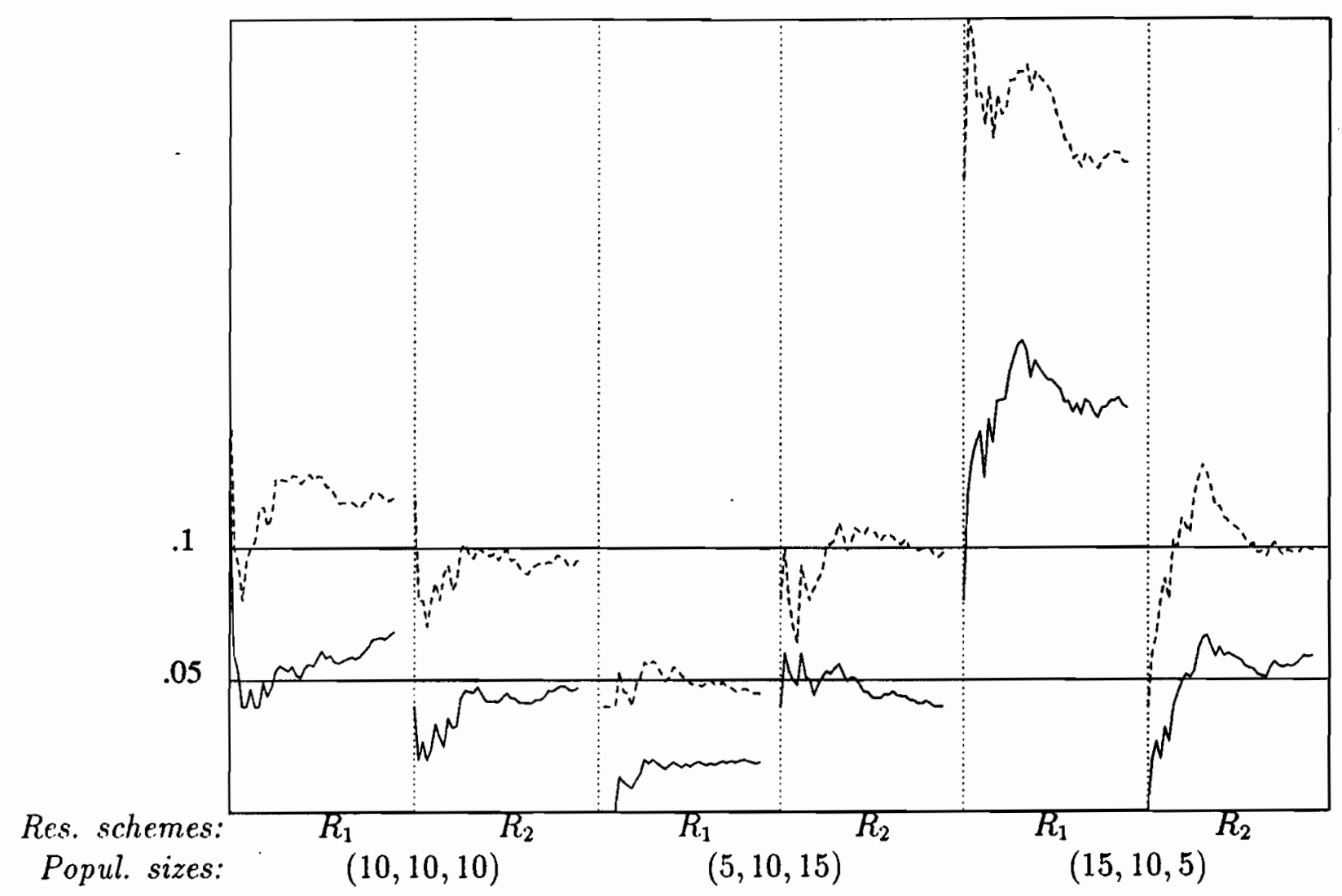

Fig. 2: Bootstrap levels with heteroscedastic data. Error distributions: $N\left(0, \sigma_{i}=i\right), i=1,2,3 . \quad-\alpha=.05, \cdots \alpha=.1$. 


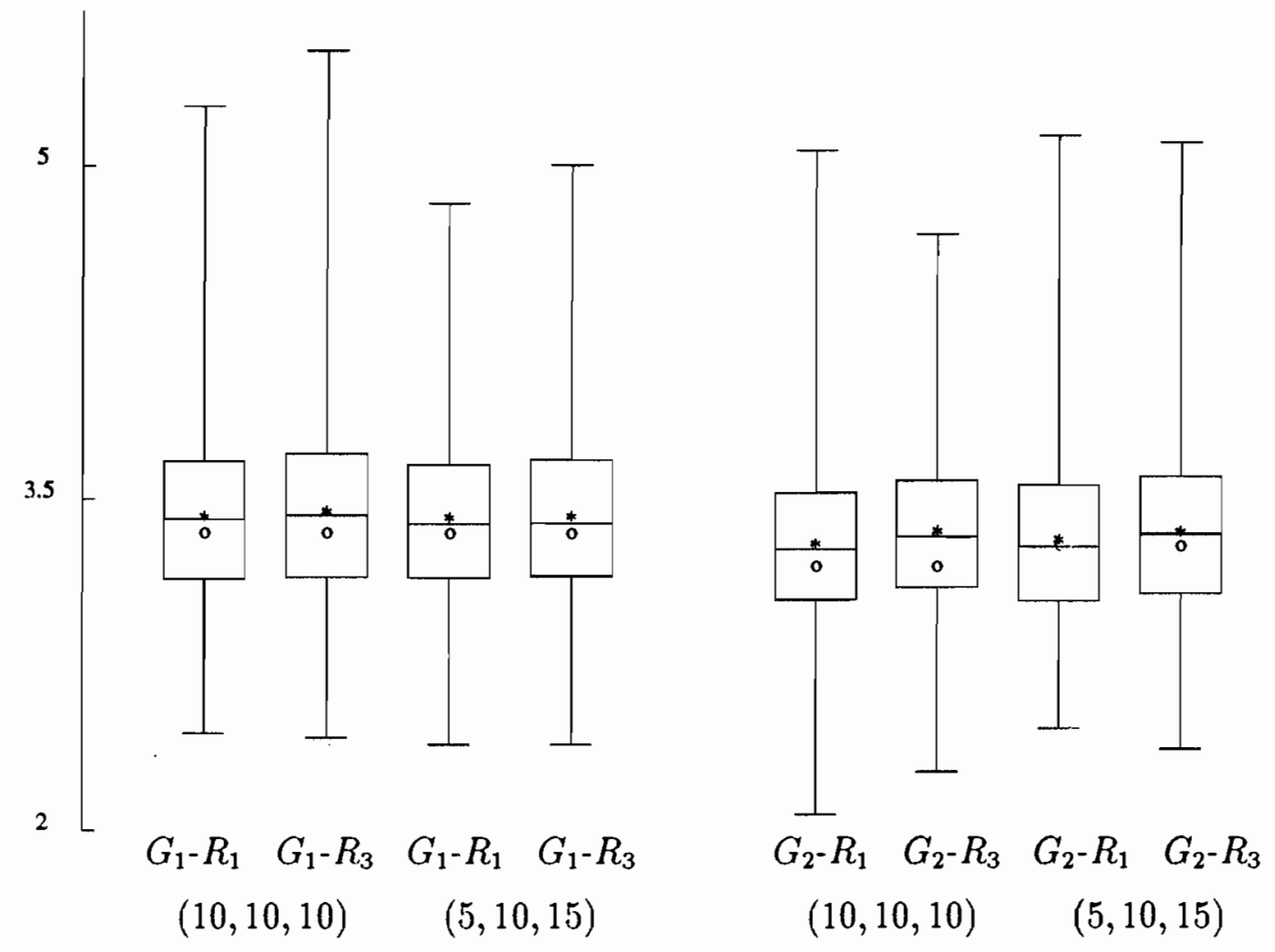

Fig. 3: Box-plots of bootstrap critical values $(\mathrm{N}=1000)$ with data from

$$
H_{A}: \mu_{1}=-1, \mu_{2}=0, \mu_{3}=1 .
$$

* Mean of bootstrap critical values. $\circ$ True critical value. 
Data from $H_{0}$

Modified F-statistic

Direct F-statistic

Data from $H_{A}$

Modified F-statistic

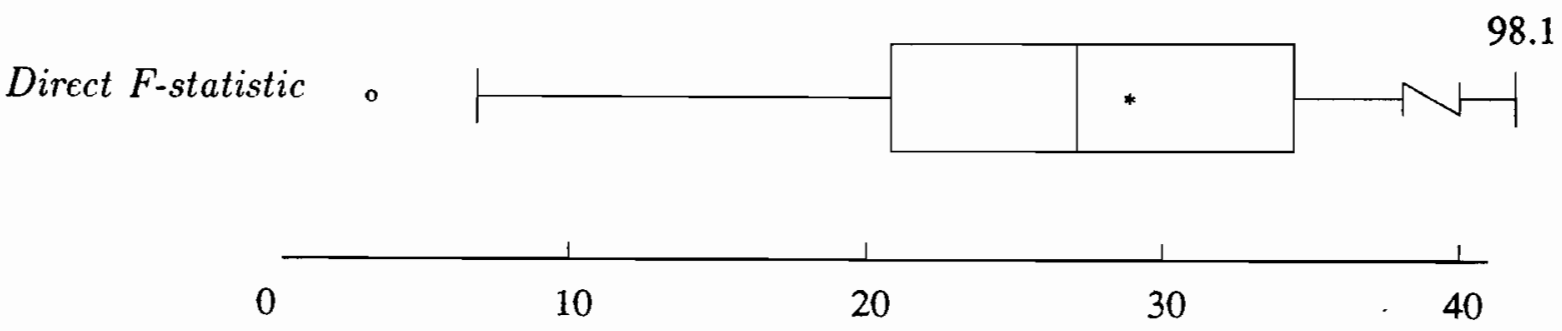

Fig. 4: Box-plots of bootstrap critical values $(N=1000)$ using modified and direct F-statistics.

* Mean of bootstrap critical values. o True critical value 
